\title{
Análisis Factorial sobre el Uso de las TIC en Instituciones Educativas de Paraguay \\ Factor Analysis on the Use of ICT in Educational Institutions in Paraguay
}

\author{
Valentina Canese ${ }^{1}$, Roberto Páez ${ }^{2}$, Jessica Amarilla ${ }^{3}$, Pamela Rodríguez ${ }^{4}$ \\ vcanese@facen.una.py, robertopaez@facen.una.py, isl-jamarilla@fil.una.py \\ ${ }^{1,2,4} \mathrm{Facultad}$ de Ciencias Exactas y Naturales \\ Universidad Nacional de Asunción \\ Asunción, Paraguay \\ ${ }^{3}$ Instituto Superior de Lenguas \\ Universidad Nacional de Asunción \\ Asunción, Paraguay
}

\begin{abstract}
Resumen- El objetivo de este trabajo es recopilar información sobre la utilización y el alcance de las TIC en el aula y ahondar conocimientos sobre la experiencia docente en la educación media en Paraguay. Es parte de un estudio más amplio, utilizando una metodología exploratoria cual-cuan por medio de un cuestionario en línea con preguntas cerradas y abiertas que consistió en 30 preguntas sobre el acceso y uso de tecnología en el aula, capacidades y capacitación docente y nivel de apoyo institucional. Se realizó un análisis estadístico descriptivo que permitió identificar el perfil de los docentes en cuanto al nivel de competencias de las TIC y el acceso limitado a los recursos tecnológicos en las instituciones de Educación Media. Además, se pudo identificar tres factores asociados a la percepción profesional de las TIC y 7 factores asociados al grado de apropiación de las mismas por medio del Análisis Factorial Exploratorio.
\end{abstract}

\section{Palabras clave: TIC - Educación Media - Paraguay}

\begin{abstract}
The objective of this study is to gather information on the use and scope of ICT in the classroom and to deepen knowledge about the teaching experience in secondary education in Paraguay. It is part of a larger study that follows an exploratory qual-quan approach by means of an online questionnaire with closed and open-ended questions consisting of 30 questions on access to and use of technology in the classroom, teacher skills and training, and level of institutional support. A descriptive statistical analysis was carried out to identify the profile of teachers in terms of the level of ICT skills and limited access to technological resources in secondary education institutions. In addition, three factors associated with the professional perception of ICT and 7 factors associated with the degree of appropriation of ICT were identified by means of Exploratory Factor Analysis.
\end{abstract}

Keywords: ICT - Secondary Education - Paraguay

\section{INTRODUCCIÓN}

La tecnología forma parte del campo de la educación desde hace muchos años y ha cambiado la forma de enfocar la enseñanza y el aprendizaje. Además, su aplicación ha dado lugar a un escrutinio en la forma en que se utiliza y cómo la experimentan los distintos agentes educativos. Consideremos primero las TIC dentro del ámbito educativo. Mahini et al., (2012) afirman que las TIC en la educación implican la planificación y facilitación del uso de computadoras para la comunicación y la enseñanza junto con otras actividades relacionadas con la educación. Sin embargo, otros han afirmado que consiste en un conjunto mucho más diverso de experiencias. Akturk y Saka (2019) hablan de una integración de la tecnología dentro de la educación a través del modelo TPACK. El uso de las TIC puede implicar el uso de cualquier dispositivo que pueda "almacenar, recuperar, manipular o transmitir información electrónicamente en forma digital" (Firmin y Genesi, 2013, p. 1606). Así también, la tecnología cumple un rol muy importante en el desarrollo de las habilidades del siglo XXI como la creatividad y la resolución de problemas (Atman Uslu y Usluel, 2019). De esta manera, los investigadores han afirmado que "la tecnología de la información y la comunicación tiene el potencial de mejorar los métodos de aprendizaje y enseñanza" (Gellerstedt et al. 2018, p. 2) y que puede crear una atmósfera centrada en el estudiante (Ahmadi, 2018), debemos ser conscientes de que es necesaria una planificación cuidadosa para que dé los resultados deseados y de que las nuevas pedagogías también deben integrarse dentro de la instrucción (Firmin y Genesi, 2013).

Hoy en día, se anima a los profesores y a las escuelas a implementar la tecnología dentro del aula. Sin embargo, parece que no existe una solución única para la correcta implementación de la tecnología (Venkatesh et al., 2016). Esto lleva a las instituciones y a los profesores a utilizar las herramientas tecnológicas de la forma que crean que mejor se adapta a las necesidades de sus alumnos. Teniendo en cuenta esto, es importante considerar la experiencia de los profesores cuando utilizan la tecnología. Por ejemplo, la opinión de los profesores sobre la tecnología hace que el aprendizaje sea más eficaz (Heitink et al., 2016), su confianza influye en el uso de la tecnología en el aula ( $\mathrm{Li}$ et al., 2018) y si ven valor en el uso y la integración de las herramientas tecnológicas podrían ser más propensos a usarlas de manera significativa y superar las barreras (Vongkulluksn et al., 2017). Sin embargo, la implementación de tecnología ha llevado a investigadores a estudiar las barreras que se presentan durante este proceso, como los recursos, los conocimientos y habilidades, así como también las actitudes sobre la tecnología por parte de los profesores (Tosuntaş et al., 2019). Así mismo, la falta de capacitación también representa un obstáculo a la hora de implementar las TIC en el proceso de enseñanza-aprendizaje (Siefert et al., 2019).

El uso de las TIC en el aula ha sido ampliamente estudiado generando información sobre diferentes modelos de implementación. Tales modelos abordan diferentes temas como la integración de las TIC y la adaptación pedagógica de la enseñanza (Akturk y Saka, 2019). Otros se enfocan en las 
características docentes y cómo estas influyen en el uso de la tecnología (Inan y Loether, 2009) así como también la percepción de valor de la tecnología para su integración en las actividades del aula (Wozney et al., 2006). Estas investigaciones responden a la necesidad de generar entendimiento sobre la experiencia del docente en cuanto a la enseñanza con TIC ya que la simple disponibilidad de herramientas tecnológicas en el aula o institución "no es suficiente para una integración significativa en un plan de estudios para profundizar el aprendizaje" (Dwyer, 2015, p. 384).

Investigaciones sobre la implementación de las TIC en el área educativa son pertinentes debido a los varios factores que afectan su uso eficiente y cómo estas herramientas aportan valor al proceso educativo. Al contemplar el panorama de experiencias docentes sobre la disponibilidad y utilización de las TIC dentro del contexto de cada institución es posible ejecutar cambios en pos de un mejor desempeño docente. Este estudio es el resultado de una investigación más amplia sobre el uso de la tecnología en las escuelas secundarias de Paraguay. El estudio busca describir las experiencias de los docentes en el uso de herramientas tecnológicas en el aula para la enseñanza de diversas asignaturas con el fin de comprender mejor las oportunidades y los desafíos que enfrentan al implementar las TIC en clase.

\section{CONTEXTO}

Las TIC se han convertido en elementos indispensables en la vida de las personas y han ganado protagonismo en el área educativa a través de los años. Sin embargo, los investigadores concuerdan que aún es necesario abordar las TIC dentro de una reforma educativa para que dé resultados claros y definitivos (Graves y Bowers, 2018).

En Paraguay, el Ministerio de Educación y Ciencias, MEC, ha generado alianzas con otras organizaciones como Itaipú Binacional, el Banco interamericano de Desarrollo y la Unión Europa para hacer frente a la falta de recursos tecnológicos, de esta manera, los esfuerzos se centraron en equipar las instituciones y capacitar a los docentes (MEC, 2015). Por otro lado, a través del Fondo Nacional de Inversión Pública y Desarrollo, FONACIDE, se implementó el programa "Mejoramiento de las condiciones de aprendizaje mediante la incorporación de TIC en establecimientos educativos y unidades de gestión educativa en Paraguay, 2015-2019” con el fin de mejorar las condiciones de aprendizaje desde la implementación de las TIC en los procesos pedagógicos y administrativos.

Los esfuerzos mencionados anteriormente son de extrema importancia ya que la educación secundaria representa la base académica para el éxito del alumnado en sus estudios superiores y universitarios. Considerando esto, las estrategias aplicadas para la mejora de la educación a través de las TIC también podrían tener un impacto positivo para el desempeño profesional del estudiante una vez se integre al mercado laboral. Debido a esto, es importante contar con información sobre el uso y el alcance de las TIC en la educación secundaria a fin de tomar decisiones en base a evidencia y aplicar soluciones eficientes que resulten en el mejoramiento de la experiencia educativa de los docentes y estudiantes.

En Paraguay existen estudios que han abordado el tema de implementación de las TIC en el aula. Por ejemplo, Prieto
(2014) se enfoca en el uso de computadoras para la inclusión social. Así también, se ha estudiado el impacto del programa con modelo $1: 1$, es decir, "Una computadora por niño" promovido por la organización no gubernamental Paraguay Educa (Sena, 2009). El estudio da a conocer sobre los cambios pedagógicos, los procesos de socialización con la tecnología, las competencias digitales de los docentes y el proceso cognitivo experimentado por los estudiantes que participaron del programa (Misiego \& Demelenne, 2015). Sin embargo, actualmente no se cuenta con estudios que aborden el uso y alcance de las TIC en la educación secundaria a modo de informar sobre las experiencias de los actores educativos sobre el tema. El objetivo de este trabajo fue recopilar información sobre la utilización y el alcance de las TIC en el aula y ahondar conocimientos sobre la experiencia docente en la educación secundaria en Paraguay con el fin de informar futuras decisiones y proponer soluciones que mejoren la educación secundaria a través del uso de herramientas tecnológicas.

\section{DESCRIPCIÓN}

El presente estudio cuenta con varias fases de recolección de datos, siendo el estudio piloto la base de este reporte. La metodología utilizada fue exploratoria cual-cuan por medio de grupos focales y un cuestionario en línea con preguntas cerradas y abiertas que fue adaptado del estudio de Wozney, Venkatesh y Abrami (2006). El cuestionario fue distribuido a través de las redes sociales que incluyó 30 preguntas referentes a la información demográfica, acceso y uso de tecnología en el aula, capacidades y capacitación docente y nivel de apoyo institucional. Participaron 117 profesores de educación secundaria de todo el Paraguay de los cuales la mayoría reside en áreas urbanas. Aquí se presentan los resultados del análisis cuantitativo. Los datos fueron analizados utilizando la planilla electrónica Excel y el software estadístico IBM SPSS 21 por medio de análisis descriptivo univariante y multivariante. Para la comparación de medias se utilizó la prueba T-student, para el contraste de igualdad de proporciones la prueba Chi-cuadrado, todos al 95\% de confianza y para el análisis multivariado se utilizó la técnica del Análisis Factorial Exploratorio.

\section{Resultados}

El cuestionario de implementación de las TIC para los docentes de la Educación Media fue aplicado en su fase de validación a 117 docentes de 14 de los 17 departamentos del país y de la capital Asunción, que corresponden al 10\% de la muestra total esperada. Con los datos recolectados se procedió a realizar el análisis de confiabilidad de los ítems incluidos con el Test Alfa de Cronbach y se obtuvo un índice de 0.951 por lo que se concluye que la escala de medición utilizada es fiable.

El 77\% de los docentes encuestados describieron que tienen un conocimiento por lo menos general de las herramientas informáticas para su uso en aula; de ellos $37 \%$ en nivel Promedio, 31\% Avanzado y 9\% Experto. Un 9\% de ellos mencionó haber intentado utilizar herramientas informáticas en sus clases y requieren capacitación continua en ellas. El 63\% de los encuestados indica que el acceso de los docentes a los recursos informáticos de la institución educativa donde laboran es aceptable, pobre o extremadamente pobre, $9 \%$ indica que es excelente (Figura 1). En cuanto al acceso de los estudiantes a los recursos tecnológicos de la institución educativa, el $77 \%$ de los docentes indica que es aceptable, pobre o extremadamente 
pobre; 4\% indicaron que es excelente (Figura 2). Ningún docente encuestado valoró como Muy Bueno o Excelente el acceso de los estudiantes de zonas rurales a las herramientas tecnológicas de la institución.

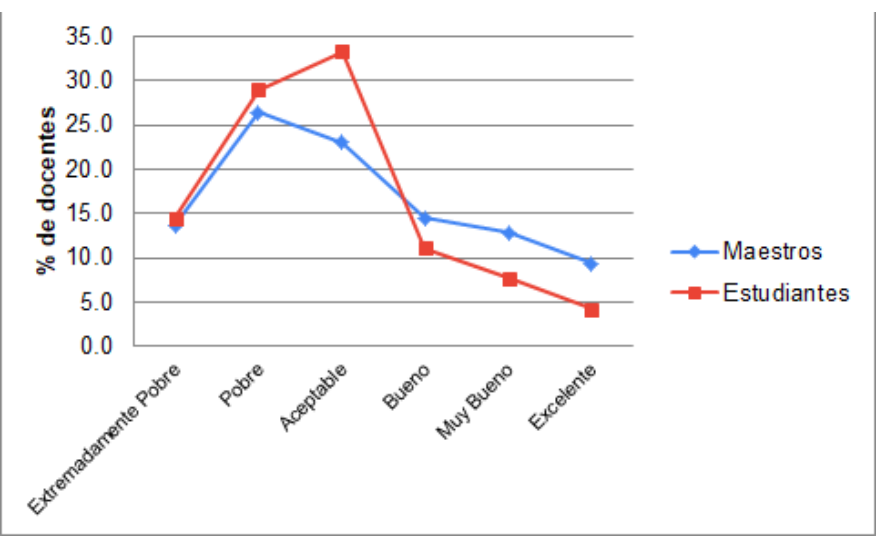

Figura 1: Acceso a los recursos informáticos de la institución educativa

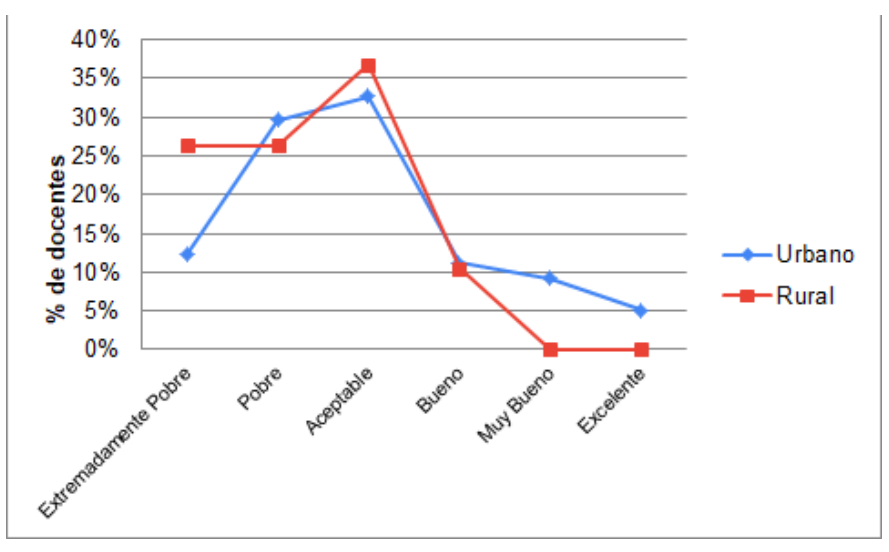

Figura 2: Acceso de los estudiantes a los recursos informáticos de la institución educativa

En cuanto al idioma empleado para el desarrollo de las clases, el $56 \%$ indicó hacerlo en lengua castellana, $41 \%$ bilingüe (Castellano - Guaraní) y lo restante en Guaraní.

Se realizó una prueba Chi-Cuadrada de igualdad de proporciones para establecer una relación entre el idioma y los niveles de apropiación de la tecnología resultando que las diferencias no son significativas al $95 \%$ de confianza ( $p$ valor $=7 \%$ ). Tampoco se pudo establecer una diferencia entre los niveles de apropiación de la tecnología por zona de residencia $(\mathrm{p}$-valor $=24 \%)$ y por Departamento de residencia ( $p$ valor $=57 \%)$.

El Cuestionario cuenta con una sección de 30 preguntas que corresponden a la opinión profesional de los docentes sobre las TIC. Con estas 30 variables se realizó un Análisis Factorial Exploratorio para identificar posibles variables latentes que permitan direccionar la investigación para su mejor interpretación en el afán de servir de apoyo para las decisiones gubernamentales en el área. Se obtuvo un coeficiente de adecuación muestral (KMO) de 0.938 lo que aseguraba que el análisis factorial se podía realizar y se distinguieron 3 factores que totalizaron un $75 \%$ de varianza total explicada.
Los 3 factores seleccionados y las relaciones más altas se dan con las siguientes variables:

Primer Factor que explica el 57\% de la variabilidad total tiene alta correlación con las siguientes variables: Motiva a los estudiantes a involucrarse más en las actividades de aprendizaje., Promueve el desarrollo de habilidades de comunicación (por ejemplo, habilidades de escritura y presentación), Ayuda a acomodar los estilos de aprendizaje personal de los estudiantes.

Segundo Factor, explica 14\% de la variabilidad total y está mayormente asociada a las variables: Exige que se dedique demasiado tiempo a problemas técnicos. Es innecesario porque los estudiantes aprenderán habilidades informáticas por su cuenta, fuera de la escuela. Requiere capacitación en habilidades de software que consumen demasiado tiempo.

El Tercer factor que explica el 4\% tiene más alta correlación con las variables: Es efectivo si los maestros participan en la selección de tecnologías informáticas para integrar., Mejora mi desarrollo profesional

También se realizó un análisis factorial exploratorio para identificar los factores asociados al grado de integración de las TIC en los procesos de enseñanza por parte de los docentes. En este caso de 34 variables asociadas se pudo identificar 7 factores que explican el $70 \%$ de la variabilidad total conjunta con una medida de adecuación muestral KMO (0.872) suficiente para la validez del procedimiento. Los 7 factores obtenidos, en orden de mayor contribución a este eje están altamente correlacionadas con variables que hacen referencia a: Requerimientos de planificación y presentación formal de documentos asociados al desarrollo de clases, Desarrollo de clases por plataformas virtuales y sus implicaciones de formación técnico-pedagógicas, Desarrollo de materiales didácticos, audiovisuales propios, Requerimientos del programa de estudios de la asignatura que se enseña, Medios de Comunicación con colegas y estudiantes, Adecuaciones Curriculares en torno al contexto actual y Promoción de la Investigación e Innovación en el aula.

La situación de Pandemia del COVID-19 fue un escenario que se tuvo en cuenta para la recolección de la información. En la Figura 3 se observa una diferencia positiva hacia los conocimientos sobre la didáctica de la educación a distancia durante la pandemia con respecto al periodo anterior a la situación sanitaria. Con un análisis de diferencia de medias pareadas T-student se pudo evidenciar diferencias significativas al 95\% de confianza (p valor prácticamente cero). 


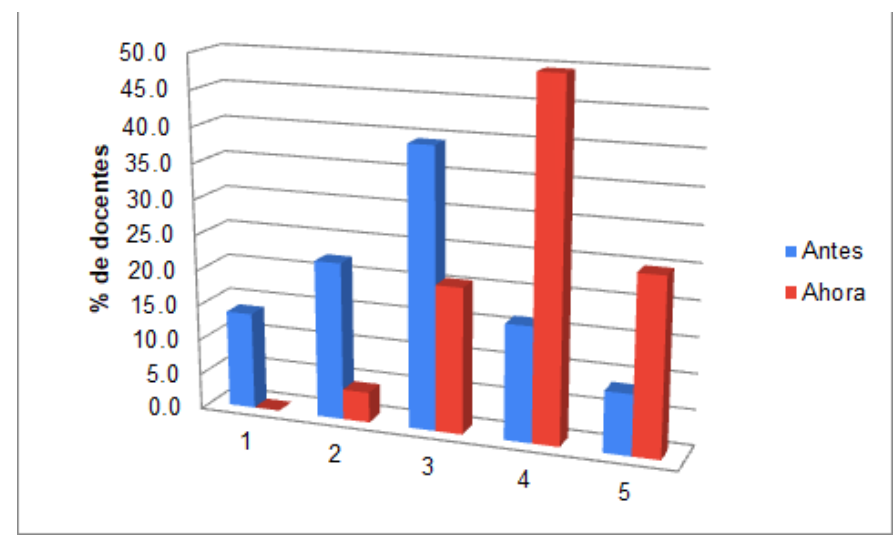

Figura 3: Grado de conocimientos sobre la Didáctica de la Educación a Distancia antes y durante la Pandemia

El proceso de integración de las TIC fue medido en una escala valorativa de 1 a 6 puntos, donde el 1 es la "percepción consciente de que la tecnología existe, pero no la he usado, tal vez incluso la estoy evitando. La posibilidad de usar computadoras. me produce ansiedad" y el 6 corresponde a la aplicación creativa "Puedo aplicar lo que sé sobre tecnología en el aula. Puedo usarlo como una ayuda de instrucción y he integrado computadoras en el plan de estudios"; al respecto el $69 \%$ de los encuestados valoraron con 5 y 6 puntos la postura de que el proceso de integración tiende a aumentar (Figura 4).

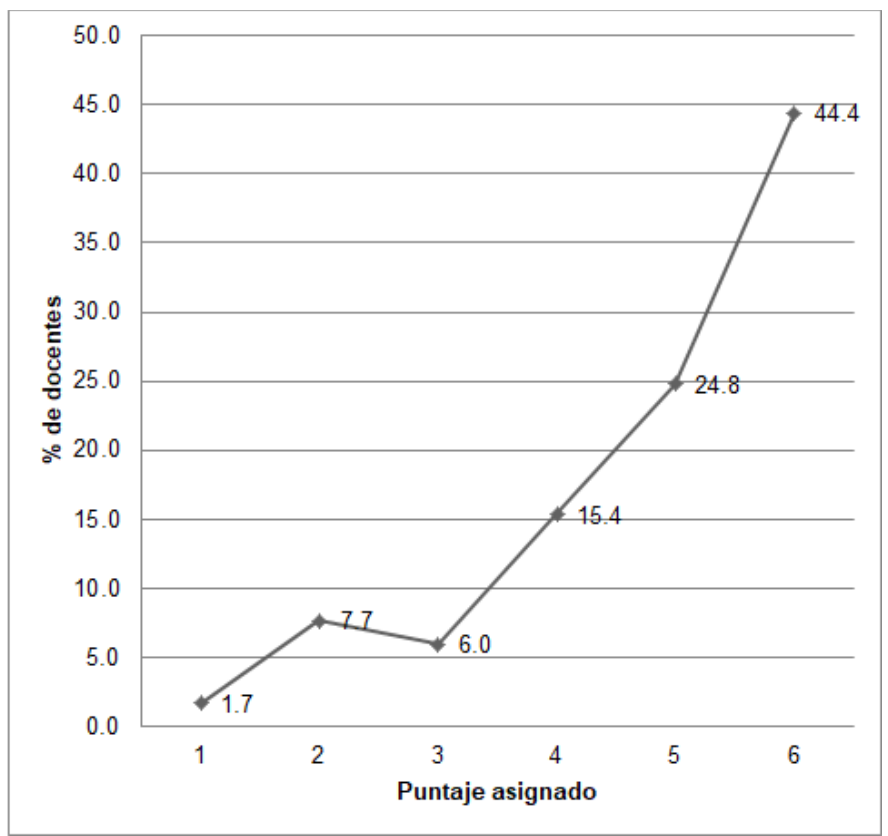

Figura 4: Valoración al proceso de integración de las TIC en el aula

Más del $80 \%$ de los docentes concluyó el cuestionario indicando tres áreas en las que desean capacitarse para mejorar su desempeño en aula con el uso de TIC: didáctica de la especialidad, estrategias de evaluación en la virtualidad y herramientas de comunicación en línea.

\section{CONCLUSIONES}

El análisis descriptivo de los datos indica que los docentes encuestados en su mayoría se reconocen con grado promedio a experto en cuanto a su nivel de competencia en el uso de herramientas tecnológicas para el desarrollo de las clases, no se registraron diferencias en estas competencias por zona geográfica de residencia o idioma que emplea en el desarrollo de las clases. La principal dificultad mencionada por los docentes, tanto para ellos como para los estudiantes es el acceso a las herramientas tecnológicas en la institución educativa donde laboran, esto se evidencia con la tendencia baja hacia la condición de excelencia en las Figuras 1 y 2.

El análisis multivariado de las opiniones profesionales de los docentes sobre las TIC permitió identificar tres factores donde es evidente que el pensamiento recurrente es hacia la mejora de las condiciones de aprendizaje de los estudiantes, Es así que del $75 \%$ explicado, $57 \%$ corresponde al factor 1 cuyas variables se relacionan con la motivación y desempeño posible de los estudiantes. El 14\% del factor 2 está relacionado a variables que mencionan las dificultades técnicas y pedagógicas de la educación mediada por tecnologías y el $4 \%$ restante, con el factor 3, se relacionan temáticas del desarrollo profesional del docente. En cuanto al grado de apropiación de las tecnologías, los docentes han indicado con sus respuestas que el Covid-19 ha sido un acelerador para la utilización de los mismos, ya que no solo se tienen en cuenta de forma didáctica para las aulas sino también para aspectos de organización de planes, estrategias de evaluación, canales de comunicación multidireccionales; respuestas que se han organizado en los 7 factores obtenidos con el método.

A partir de este estudio preliminar, se realizará una muestra probabilística de todos los docentes del nivel medio de Paraguay para medir el uso y apropiación de las TIC en las instituciones educativas oficiales. De esta manera se espera contribuir a entender mejor cuáles son los principales factores que inciden en el uso de las TIC por parte de los docentes, principales agentes educativos. A partir de estos resultados se espera informar a la política pública para que se puedan tomar decisiones que ayuden a potenciar el papel de las TIC en la educación y a asegurar el acceso y aprovechamiento de estas herramientas.

\section{AgRADECIMIENTOS}

Este estudio es realizado con apoyo de CONACYT, Paraguay (PINV18-121). El contenido de la misma es responsabilidad exclusiva de los autores y en ningún caso se debe considerar que refleja la opinión del CONACYT. Agradecemos además a todos los participantes de este estudio.

\section{REFERENCIAS}

Ahmadi D M R. (2018) The Use of Technology in English Language Learning: A Literature Review. IJREE; 3(2). 112-125. URL: http://ijreeonline.com/article-1-120en.html

Akturk, A.O. \& Saka Ozturk, H. (2019). Teachers' TPACK levels and students' self-efficacy as predictors of students' academic achievement. International Journal of Research in Education and Science (IJRES), 5(1), 283-294. 
Atman Uslu, N., \& Usluel, Y. K. (2019). Predicting technology integration based on a conceptual framework for ICT use in education. Technology, Pedagogy and Education, 28(5), 517-531. doi: 10.1080/1475939X.2019.1668293

Dwyer, B. (2015). Engaging All Students in Internet Research and Inquiry. The Reading Teacher, 69(4), 383-389. doi: $10.1002 /$ trtr.1435

Firmin, M. W., \& Genesi, D. J. (2013). History and implementation of classroom technology. Procedia Social and Behavioral Sciences, 93, 1603-1617. doi: 10.1016/j.sbspro.2013.10.089

Gellerstedt, M., Babaheidari, S. M., \& Svensson, L. (2018). A first step towards a model for teachers' adoption of ICT pedagogy in schools. Heliyon, 4(9), e00786. doi: 10.1016/j.heliyon.2018.e00786

Graves, K. E., \& Bowers, A. J. (2018). Toward a typology of technology-using teachers: A latent class analysis (Lca) of the nces fast response survey system teachers' use of educational technology in U.S. Public schools, 2009(FRSS 95). doi: 10.7916/D8-DDMV-Y558

Heitink, M., Voogt, J., Verplanken, L., van Braak, J., \& Fisser, P. (2016). Teachers' professional reasoning about their pedagogical use of technology. Computers \& Education, 101, 70-83. doi: 10.1016/j.compedu.2016.05.009

Inan, F. A., \& Lowther, D. L. (2009). Factors affecting technology integration in K-12 classrooms: a path model. Educational Technology Research and Development, 58(2), 137-154. doi: 10.1007/s11423-009-9132-y

Mahini, F., Forushan, Z. J.-A., \& Haghani, F. (2012). The Importance of Teacher's Role in Technology-Based Education. Procedia - Social and Behavioral Sciences, 46, 1614-1618. doi:10.1016/j.sbspro.2012.05.348

MEC (2015). Mejoramiento de las condiciones de aprendizaje mediante la incorporación de TIC en establecimientos educativos y unidades de gestión educativa, en Paraguay. Asunción, Paraguay: Ministerio de Educación y Cultura

Misiego P., \& Demelenne D. (2015). Las prácticas pedagógicas y la incorporación de la computadora en el aula: una experiencia desde el programa "Una computadora por niño"(Paraguay). Perspectiva Educacional, 54(1). 131148 doi: 10.4151/07189729-Vol.54-Iss.1-Art.169

Prieto, O. M. G. (2014). Computadoras para la Inclusión Social en el Paraguay. FPUNE Scientific, 4(4).

Sena E., (2009). Inclusión Digital en Paraguay. ¿Utopía o Realidad?. II Conferencia Internacional sobre Brecha Digital e Inclusión Social. Leganés, Madrid. Recuperado de https://core.ac.uk/download/pdf/30044909.pdf

Siefert, B., Kelly, K., Yearta, L., \& Oliveira, T. (2019). Teacher Perceptions and Use of Technology Across Content Areas with Linguistically Diverse Middle School Students. Journal of Digital Learning in Teacher Education, 1-15. doi: $10.1080 / 21532974.2019 .1568327$

Tosuntaş, Ş. B., Çubukçu, Z., \& İnci, T. (2019). A holistic view to barriers to technology integration in education. Turkish Online Journal of Qualitative Inquiry, 349-371. doi:10.17569/tojqi.613969
Venkatesh, V., Rabah, J., Fusaro, M., Couture, A., Varela, W., \& Alexander, K. (2016). Factors impacting university instructors' and students' perceptions of course effectiveness and technology integration in the age of web 2. 0. McGill Journal of Education, 51(1), 533-561. doi: $0.7202 / 1037358 \mathrm{ar}$

Vongkulluksn, V. W., Xie, K., \& Bowman, M. A. (2017). The role of value on teachers' internalization of external barriers and externalization of personal beliefs for classroom technology integration. Computers \& Education, $\quad 118, \quad 70-81 . \quad$ doi: 10.1016/j.compedu.2017.11.009

Wozney, L., Venkatesh, V., \& Abrami, P. (2006). Implementing computer technologies: Teachers' perceptions and practices. Journal of Technology and teacher education, 14(1), 173-207. 